\title{
Wrist Dorsal Sensor Array for Finger Movement Recognition
}

\author{
Yaohui Hu, ${ }^{1,2}$ Yadong Chen, ${ }^{1,2,3}$ Xiaobin Yi, ${ }^{4}$ Jiquan Zhong, ${ }^{1,2,5}$ Xing Wang, $, 2,5$ \\ Zhiyong Yao, ${ }^{1,2,3}$ Yingzhao $\mathrm{Hu},{ }^{1,2,3}$ Tao Zhang, ${ }^{6}$ and Yong Fang ${ }^{1,2^{*}}$ \\ ${ }^{1}$ National Engineering Laboratory of Special Display Technology, Hefei University of Technology, \\ Hefei 230009, China \\ ${ }^{2}$ Academy of Opto-electronics Technology, Hefei University of Technology, Hefei 230009, China \\ ${ }^{3}$ School of Instrument Science and Opto-electronics Engineering, Hefei University of Technology, \\ Hefei 230009, China \\ ${ }^{4}$ Department of Physics and Electronic Information Engineering, Qinghai Normal University, \\ Xining 810000, China \\ ${ }^{5}$ School of Electronic Science and Applied Physics, Hefei University of Technology, \\ Hefei 230009, China \\ ${ }^{6}$ Hefei Stream Opto-electronics Technology Co., Ltd., Hefei 230009, China
}

(Received April 22, 2021; accepted July 7, 2021)

Keywords: PVDF sensor, wrist dorsal, human-computer interaction, wearable sensor, finger movement

$\mathrm{Hu}$ proposed a method that recognizes finger movements by detecting wrist muscles for human-computer interaction (HCI). Considering human habits and aesthetics, the sensor is placed on the back of the wrist. We first designed a polyvinylidene fluoride (PVDF) piezoelectric thin-film sensor unit with a planar elastic substrate. By studying the effects of the hardness and thickness of the substrate, we designed the sensor unit to have a Shore-A hardness of 23 and a thickness of $2 \mathrm{~mm}$. Then we constructed a $4 \times 2$ sensor matrix with a size of $25 \times 15 \mathrm{~mm}^{2}$. To build a finger movement dataset, we collected wrist dorsal movement signals when the fingers moved using the sensor matrix. Then we used a four-layer back-propagation (BP) neural network to recognize the finger movements. We experimentally demonstrated that even on the dorsal wrist side, finger movements could be recognized. The recognition rate of the general model using mixed personal data was $79 \%$. In comparison, the recognition rate of the individual model using personal data was $94 \%$.

\section{Introduction}

$\mathrm{Hu}$ et al. and Zhang et al. proposed a method ${ }^{(1,2)}$ based on sensor to recognize finger movements by detecting muscles around the wrist for human-computer interaction (HCI). One of the key technologies for HCI is movement recognition based on sensors, such as surface electromyography $(\mathrm{SEMG})^{(3,4)}$ methods based on force-sensitive resistance (FSR), ${ }^{(5,6)}$ a ceramic piezoelectric sensor (CPZS), ${ }^{(7)}$ and a capacitive pressure sensor (CPS), ${ }^{(8)}$ and novel methods based on a flexible epidermal tactile sensor array (FETSA), ${ }^{(9)}$ a modified barometric pressure sensor (MBPS), ${ }^{(10)}$ photoplethysmographic $(\mathrm{PPG}),{ }^{(11)}$ and electrical impedance tomography (EIT). ${ }^{(12)}$

*Corresponding author: e-mail: fy9903@hfut.edu.cn https://doi.org/10.18494/SAM.2021.3372 
For a wearable device employed for HCI, the most important feature is the accuracy of recognition. Therefore, we believe that the model and algorithm for HCI can be further improved. We propose the following optimizations. First, we reduce the number of recognized actions and focus only on the finger movements. Second, we stipulate that the hand can only operate on a particular surface to reduce the number of degrees of freedom (DOFs) of hand movement, assuming that people will get used to the operation mode, in the same way that they operate a computer mouse on a specific surface. When such a surface is used, they are less likely to suffer from wrist pain after long-term operation involving the selection and movement of small objects on a surface.

In this paper, we proposed a polyvinylidene fluoride (PVDF) sensor unit and a PVDF sensor matrix that can be placed under a dial. Then we used this sensor matrix to collect the wrist dorsal movement signal when fingers move. Then we used a back-propagation (BP) neural network to train and recognize the finger movements. Our aims are to develop a general model to fit all wrists and to shorten the training time of individual models.

The finger movement recognition method proposed in this paper is based on a PVDF pressure sensor on the dorsal side of the wrist. It is different from other existing methods based on pressure sensors (such as CPS and CPZS). As reported in this paper, we studied the feasibility of finger movement recognition with the sensor located on the dorsal side of the wrist, which is one of the key issues in successfully applying this method to wristwatch-type wearable devices, and we obtained good results. Compared with SEMG, another wrist sensor used for gesture recognition, the PVDF sensor discussed in this paper is more applicable for general use. Moreover, it does not have special requirements for the wearer, such as the electrode should be close to the skin, and both the skin and the electrode need to be coated with conductive glue.

\section{Materials and Methods}

PVDF has the advantages of high sensitivity, a high response speed, and a wide frequency band. A typical PVDF piezoelectric film with an electrode-PVDF film-electrode sandwich structure is shown in Fig. 1.

PVDF films usually work in two modes: the 31-mode and 33-mode. ${ }^{(13)}$ When a PVDF film is fixed on an elastic material, it works in the 31-mode. We refer to this elastic material as the substrate. As shown in Fig. 1, when a vertical force $F(\mathrm{~N})$ is pressed on the PVDF film and substrate, the PVDF film produces strain $s$ in the $x$ and $y$ directions. The electric charge of the PVDF film has the following expression:

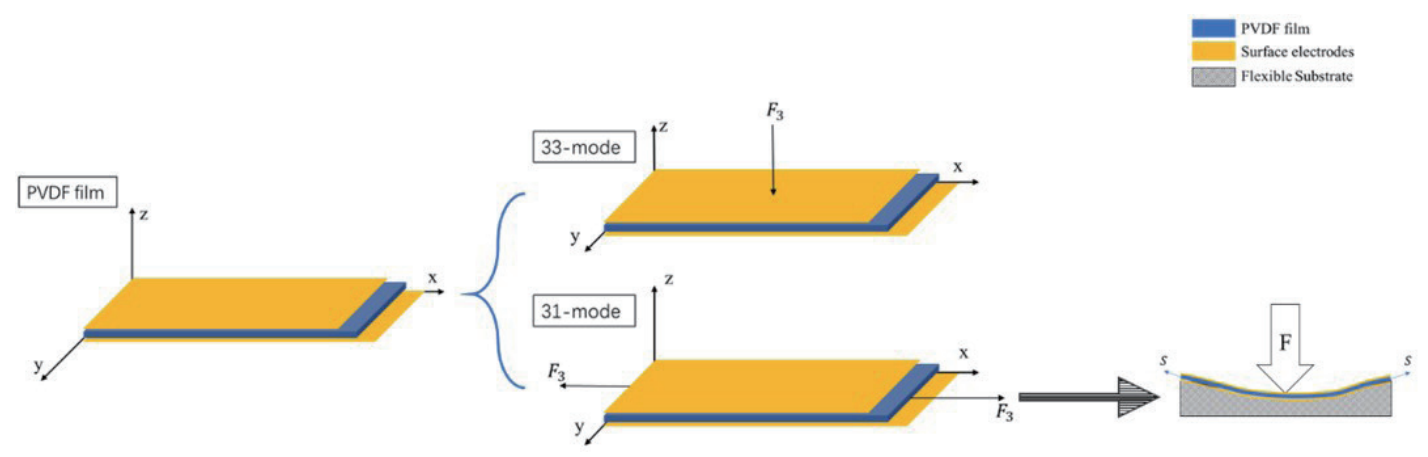

Fig. 1. (Color online) Typical PVDF piezoelectric film. 


$$
Q=\left(d_{x} \varepsilon_{x}+d_{y} \varepsilon_{y}\right) E A,
$$

where $d_{x}$ and $d_{y}$ are the piezoelectric coefficients of the PVDF film in the $x$ and $y$ directions, $\varepsilon_{x}(\mathrm{~m})$ and $\varepsilon_{y}(\mathrm{~m})$ are the deformations of the PVDF film in the $x$ and $y$ directions, respectively, $E(\mathrm{~Pa})$ is the elastic modulus of the PVDF, $A\left(\mathrm{~m}^{2}\right)$ is the area of the PVDF film surface electrode, and $Q(\mathrm{C})$ is the output charge of PVDF film.

Because the PVDF film is fixed on the substrate, the deformations $\varepsilon_{x}$ and $\varepsilon_{y}$ of the PVDF film are related to the properties of the substrate. For substrates with the same force and thickness, a softer substrate produces a larger output charge $Q$. Also, for substrates with the same hardness, a thinner substrate provides a smaller range of charge $Q$.

\subsection{Sensor configuration}

The parameters of the PVDF film are shown in Table 1. We designed a PVDF sensor unit and matrix with a planar backing configuration as shown in Fig. 2.

Finger movements involve many wrist muscle and tendon movements. ${ }^{(14)}$ To obtain the highest resolution along the circumference of the back of the wrist, we designed a $4 \times 2$ sensor matrix as shown in Fig. 2.

\subsection{Backing modulation}

A Shore durometer is used for the Shore-A hardness measurement of materials such as rubbers, elastomers, and polymers. To study the effect of the substrate material hardness and thickness on the pressure-voltage response curve of the sensor unit, we selected silicone rubber with Shore-A hardnesses of 23 and 53 as the materials of the substrate. We prepared five different sensor units for testing, as listed in Table 2, where $0 \mathrm{~mm} / 99$ denotes the sensor without a substrate.

Table 1

PVDF film parameters.

\begin{tabular}{lc}
\hline Thickness $(\mathrm{m})$ & $5 \times 10^{-5}$ \\
\hline$E(\mathrm{~Pa})$ & $2.5 \times 10^{9}$ \\
\hline$d_{x}$ & $17 \times 10^{-12}$ \\
\hline$d_{y}$ & $3 \times 10^{-12}$ \\
\hline$\varepsilon / \varepsilon_{0}$ & $9.5 \pm 1.0$ \\
\hline
\end{tabular}

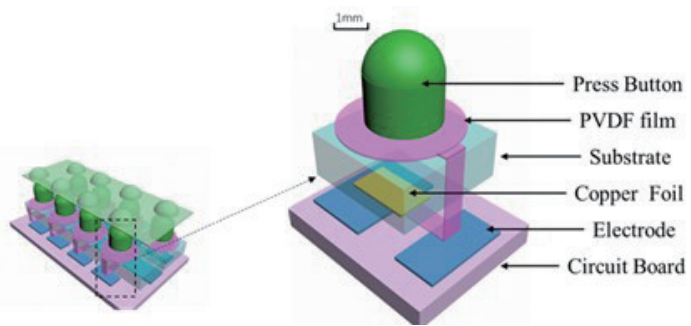

Fig. 2. (Color online) Sensor unit and matrix configuration.

Table 2

Substrate of PVDF sensor with different thickness/hardness.

\begin{tabular}{ccccc}
\hline \multicolumn{5}{c}{ Thickness $(\mathrm{mm}) /$ Hardness (Shore-A) } \\
\hline $2 \mathrm{~mm} / 23$ & $1 \mathrm{~mm} / 23$ & $2 \mathrm{~mm} / 53$ & $1 \mathrm{~mm} / 53$ & $0 \mathrm{~mm} / 99$ \\
\hline
\end{tabular}


The impulse signal is the standard test signal for sensors. In this study, we used an electromagnetic coil modified from a loudspeaker coil, which was controlled by a $1 \mathrm{~Hz}, 1 \%$ duty pulse signal, to simulate the impulse signal. The experimental device and its schematic diagram are shown in Fig. 3.

We used this device to test the sensors listed in Table 2. The response signal (yellow line) of the sensor unit is shown in Fig. 4 when the Shore-A hardness is 23, the substrate thickness is $2 \mathrm{~mm}$, and the power supply (green line) is $1 \mathrm{~V}$.

Each group of PVDF sensor units listed in Table 2 was tested using the above device. We considered the peak voltage of the response signal as the output voltage of the PVDF sensor. The substrate pressure-voltage response curve of each unit is shown in Fig. 5.

For the same thickness and pressure, we obtained a lower output voltage of the sensor with a harder substrate. For the same hardness, we obtained a greater output voltage of the sensor unit with a thicker substrate.

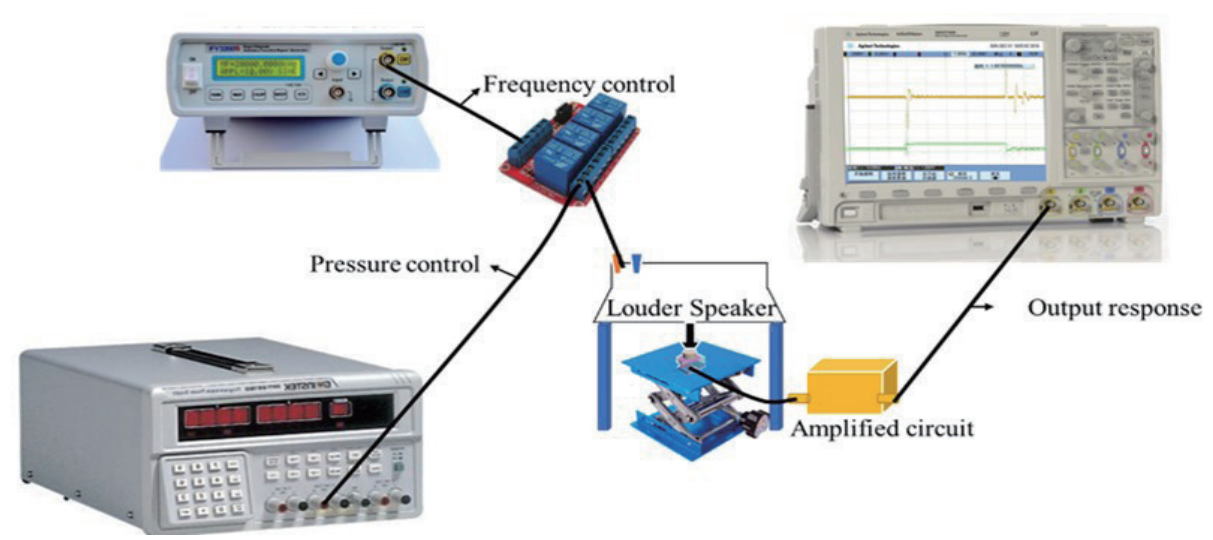

Fig. 3. (Color online) Experimental device and schematic diagram.

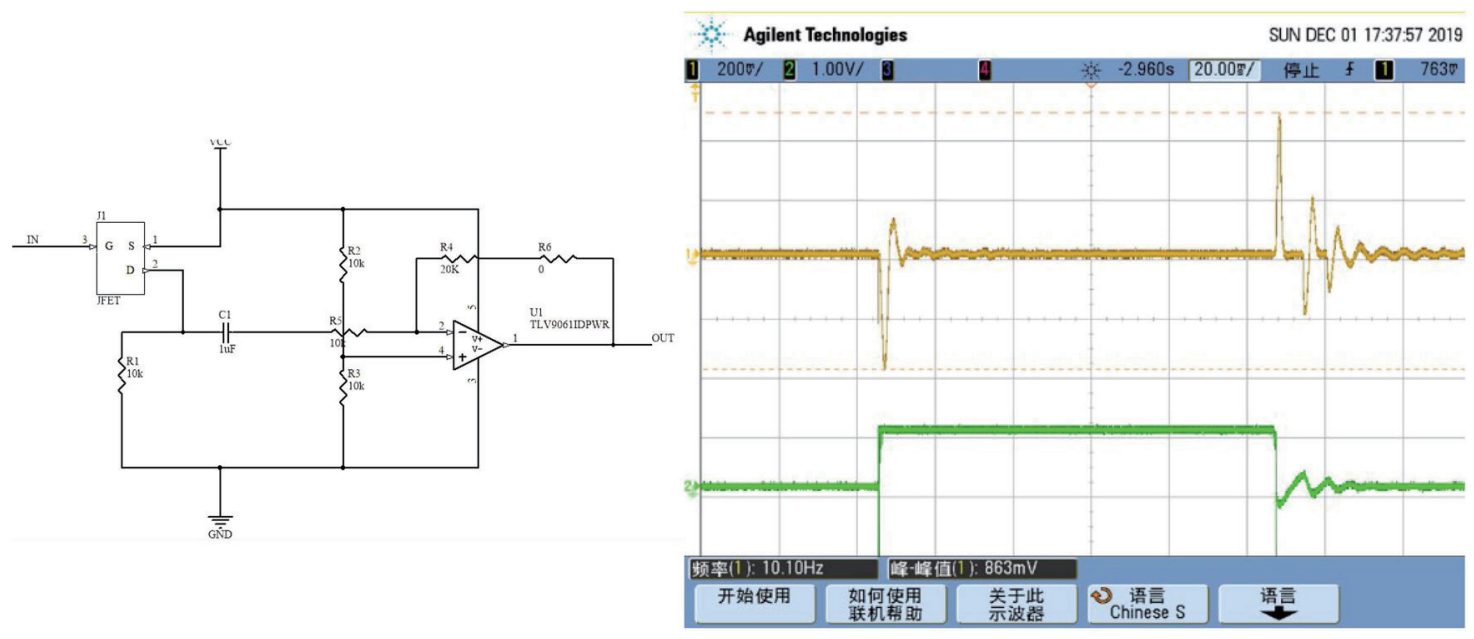

Fig. 4. (Color online) Amplified circuit diagram and waveform diagram. 


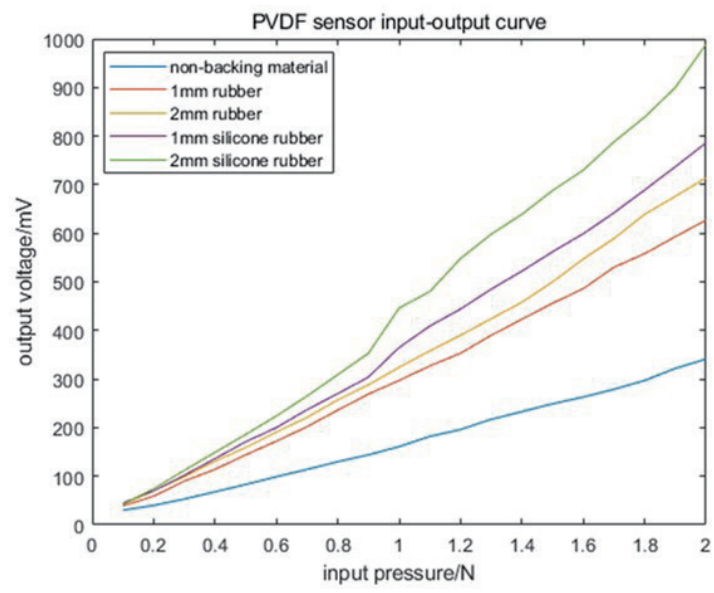

Fig. 5. (Color online) Sensor response curve with different substrates: $2 \mathrm{~mm}$ silicone rubber exhibited the greatest output voltage.

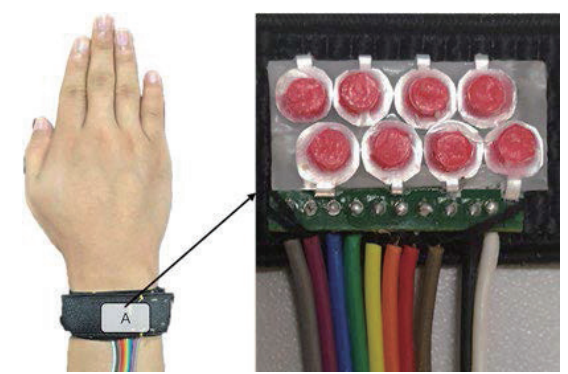

Fig. 6. (Color online) Sensor and application.

By considering the characteristics of dorsal wrist movement, ${ }^{(15)}$ we attempted to obtain a more sensitive sensor. We selected the substrate with a Shore-A hardness of 23 and a thickness of $2 \mathrm{~mm}$ and we fabricated the sensor matrix using the substrate, as shown in Fig. 6.

\section{Experimental Results}

We used the sensor matrix to collect three types of basic movement: index finger clicks, middle finger clicks, and hand moves. Four subjects participated in our experiment and more than 1000 samples were collected for each subject. All subjects agreed with the data collection and experiment approval. Each sample contained $8 \times 64$ bits and the sampling rate was $1000 \mathrm{~Hz}$. We used a four-layer BP neural network (BPNN) to recognize the finger movements. The BPNN input layer had 512 neurons, the output layer had three neurons, and the hidden layer are both set to $100 \times 2$.

As shown in Fig. 7, the recognition rate for each person was above 94\%, which shows that the sensor matrix with the neural network could effectively detect and recognize the finger movements. In contrast, the recognition rate for the general model using the mixed personal data of the four subjects was only $79 \%$.

\section{Discussion and Conclusions}

As shown in Table 3, similar to the results obtained by the PVDF* method in this paper, the SEMG method, which also collected myoelectric signals on the dorsal side of the wrist, recognized 3-5 types of gestures and its recognition rates are $96.7 \%$ and $81.9 \%$.

The gesture recognition methods based on mechanical sensing usually collect signals on the inner wrist or around the wrist, where the gesture motion signals change drastically and are easy to detect, and have achieved recognition rates of more than $90 \%$. 


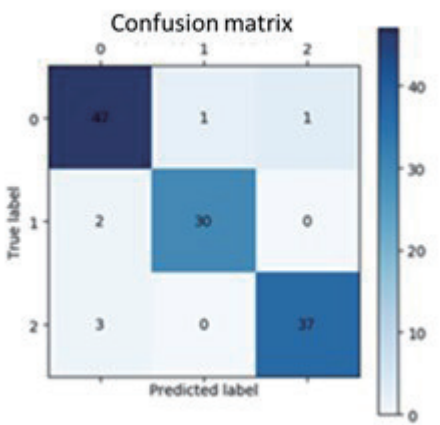

(a)

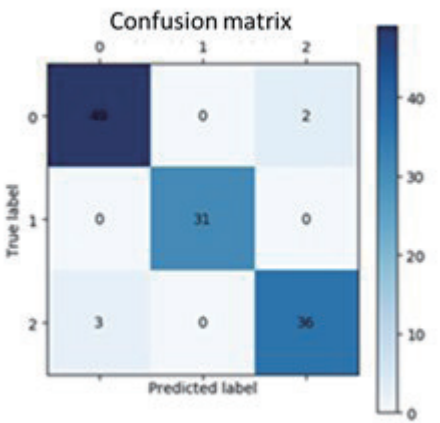

(c)

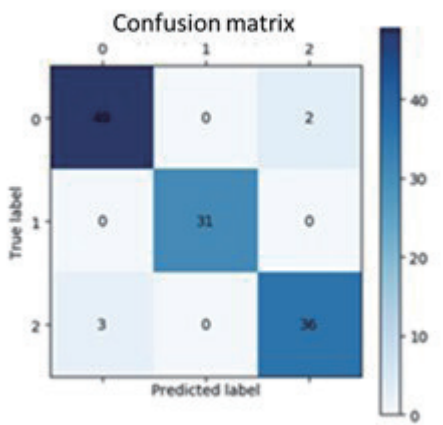

(b)

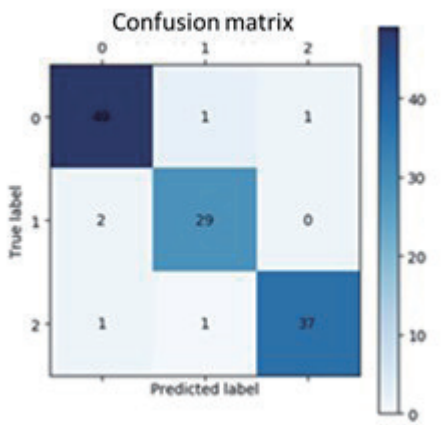

(d)

Fig. 7. (Color online) The four individual subjects' recognition rates of three movements are up to $94 \%$. (a) Subject 1: 94.24\%. (b) Subject 2: 95.96\%. (c) Subject 3: 95.96\%. (d) Subject 4: 95.04\%.

Table 3

Experimental conditions and recognition results of different sensors.

\begin{tabular}{lccccc}
\hline Sensor & Recognition rate & Signal source & Sensor quantity & Movement quantity & Sensor position \\
\hline SEMG & $94.7 \% / 87.9 \%$ & Myoelectric signal & 3 & $3 / 5$ & Dorsal wrist \\
\hline CPZS & $97 \%$ & $\begin{array}{c}\text { Muscle pressure signal } \\
\text { (MPS) }\end{array}$ & 6 & 5 & Inner wrist \\
\hline CPS & $>90 \%$ & MPS & 3 & 3 & Inner wrist \\
\hline MBPS & $51-94 \%$ & MPS & $1-10$ & 15 & Inner wrist \\
\hline FETSA & $95.75 \%$ & MPS & 16 & 5 & Around wrist \\
\hline PVDF & $97 \%$ & MPS & 8 & 3 & Around wrist \\
\hline PVDF $^{*}$ & $94 \% / 79 \%$ & MPS & 8 & 3 & Dorsal wrist
\end{tabular}

The placement of the pressure sensor is an important factor that affects the recognition accuracy. Considering the usage habits and aesthetics, we placed the sensor on the dorsal side of the wrist. In addition, in view of the weak signals obtained from the dorsal side of the wrist, we proposed a PVDF sensor unit with a planar elastic substrate. By adjusting the hardness and thickness of the substrate, the sensor can both be sensitive to weak motion and have a linear response in the entire range of motion amplitude. Using the above-mentioned sensor unit array and BP algorithm, we obtained a recognition rate of $94 \%$. It can be seen from Table 3 that the recognition rate was only $3 \%$ less than those at other positions. However, the feasibility of using the PVDF sensor array to recognize mouse movements on the dorsal side of the wrist has been demonstrated. 


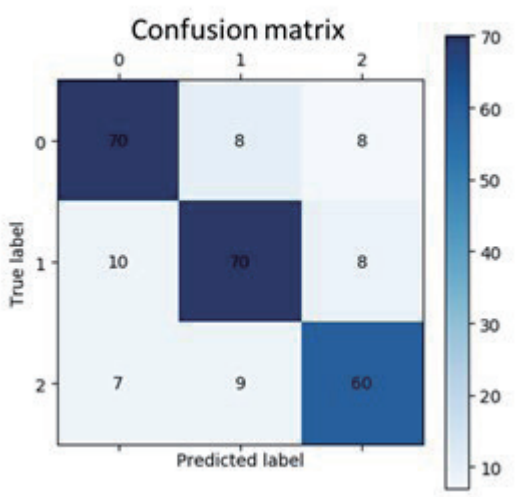

Fig. 8. (Color online) The recognition rate of the general model to fit everyone's wrist is $79 \%$.

As is shown in Fig. 8, when training the general model, the recognition rate was 79\%, which is much lower than the recognition rate for each person. In this study, we used the simplest neural network algorithm for the task of recognition, and the use of complex neural networks or deep learning algorithms might improve the recognition rate.

\section{References}

1 Y. H. Hu, W. W. Kang, Y. Fang, L. R. Xie, L. Z. Qiu, and T. Jin: Appl. Sci. 8 (2018) 836. https://doi.org/ ARTN83610.3390/app8050836

2 Y. F. Zhang, B. Liu, Z. Q. Liu, J. Y. Huang, and R. Sun: 2019 IEEE 16th Int. Conf. Wearable and Implantable Body Sensor Networks (Bsn) (2019). https://doi.org/10.1109/BSN.2019.8771036

3 S. M. Mane, R. A. Kambli, F. S. Kazi, and N. M. Singh: Proceedings of 4th Int. Conf. Advances in Computing, Communication and Control (Icac3'15) 49 (2015) 58. https://doi.org/10.1016/j.procs.2015.04.227

4 J. He and N. Jiang: Front. Bioeng. Biotechnol. 8 (2020) 58. https://doi.org/10.3389/fbioe.2020.00058

5 Y. F. Zhang, B. Liu, and Z. Q. Liu: 2018 IEEE 15th Int. Conf. Biomedical and Health Informatics (Bhi) and the Wearable and Implantable Body Sensor Networks (Bsn) 2018-Janua (2018) 177. https://doi.org/10.1109/ BSN.2018.8329687

6 A. Dementyev and J. A. Paradiso: UIST 2014 - Proceedings of the 27th Annual ACM Symposium on User Interface Software and Technology (2014) 161. https://doi.org/10.1145/2642918.2647396

7 R. Booth and P. Goldsmith: J. Med. Biol. Eng. 38 (2018) 284. https://doi.org/10.1007/s40846-017-0303-8

8 X. P. Liang, R. Ghannam, and H. Heidari: IEEE Sensors Journal 19 (2019) 1082. https://doi.org/10.1109/ Jsen.2018.2880194

9 S. W. Byun and S. P. Lee: J. Electr. Eng. Technol. 13 (2018) 1731. https://doi.org/10.5370/Jeet.2018.13.4.1731

10 P. B. Shull, S. Jiang, Y. Zhu, and X. Zhu: IEEE Trans. Neural. Syst. Rehabil. Eng. 27 (2019) 724. https://doi. org/10.1109/TNSRE.2019.2905658

11 T. M. Zhao, J. Liu, Y. Wang, H. B. Liu, and Y. Y. Chen: IEEE Conf. Computer Communications (IEEE Infocom 2018) 2018-April (2018) 1457. https://doi.org/10.1109/INFOCOM.2018.8486006

12 Y. Wu, D. Jiang, J. F. Duan, X. Liu, R. Bayford, and A. Demosthenous: 2018 IEEE Int. Symposium on Circuits and Systems (Iscas) 2018-May (2018) 1. https://doi.org/10.1109/ISCAS.2018.8351296

13 W. Cao and W. Yu: IOP Conference Series: Earth Environ. Sci. 170 (2018). https://doi.org/10.1088/17551315/170/4/042156

14 A. K. Garland, D. S. Shah, and A. E. Kedgley: J. Biomech. 68 (2018) 136. https://doi.org/10.1016/j. jbiomech.2017.12.024

15 Y. H. Hu, L. R. Xie, Y. D. Chen, K. He, Y. Fang, W. W. Kang, Z. X. Yao, and G. Q. Fang: Sens. Mater. 31 (2019) 2931. https://doi.org/10.18494/Sam.2019.2444 


\section{About the Authors}

Yaohui Hu received his B.S. degree from Hefei University of Technology, China, in 1992 and his M.S. and Ph.D. degrees from the University of Science and Technology of China in 1999 and 2002, respectively. From 2003 to 2005, he was a lecturer at the University of Science and Technology of China. From 2005 to 2007, he was a lecturer at Hefei University of Technology. Since 2008, he has been an associate professor at Hefei University of Technology. His research interests include biomedical engineering and human-computer interaction technology.

Yadong Chen received his B.S. degree from Wuhan University of Technology, China, in 2017. He is currently pursuing his M.S. degree in the Department of Biomedical Engineering, School of Instrument Science and Opto-electronics Engineering, Hefei University of Technology, China. His research interests include human-machine interaction, biomedical engineering, sensors, and control engineering.

Xiaobin Yi received his B.S. degree in applied physics from Hefei University of Technology, Hefei, China, in 1992 and his M.S. degree in physics from Lanzhou University, Lanzhou, China, in 2005. He is presently vice professor at the Department of Physics and Electronic Information Engineering, Qinghai Normal University, Xining, Qinghai, China. His research interests is intelligent sensor technology.

Jiquan Zhong received his B.S. degree from Hefei University in 2019. He is currently pursuing his M.S. degree at the School of Electronic Science and Applied Physics, Hefei University of Technology, China. His current research interests include intelligent signal processing and applications of biosensors.

Xing Wang received his B.S. degree from Henan Polytechnic University, China, in 2018. He is currently pursuing his M.S. degree at the School of Electronic Science and Applied Physics, Hefei University of Technology, China. His research interests include human-machine interaction, software programming, and sensors.

Zhiyong Yao received his B.S. degree from Hefei University of Technology, China, in 2020. He is currently pursuing his M.S. degree at the Department of Biomedical Engineering, School of Instrument Science and Opto-electronics Engineering, Hefei University of Technology, China. His research interests include human-machine interaction, biomedical engineering, and image processing.

Yingzhao Hu received his B.S. degree from Anhui Xinhua University, China, in 2020. He is currently pursuing his M.S. degree at the Department of Electronic Information, School of Instrument Science and Opto-electronics Engineering, Hefei University of Technology, China. His research interests include sensors and mechanical drawing. 
Tao Zhang received his B.S. degree from Anhui University, China, in 1997 and his M.S. and Ph.D. degrees from Anhui Institute of Optics and Fine Mechanics, Chinese Academy of Sciences, China, in 2000 and 2003, respectively. His research interests include virtual reality and human-computer interaction technology.

Yong Fang received his B.S., M.S., and Ph.D. degrees from Hefei University of Technology, China, in 2003, 2007, and 2017, respectively. He is an assistant professor at Hefei University of Technology. His research interests include photoelectric information technology and sensors. 